PROCEEDINGS OF THE

AMERICAN MATHEMATICAL SOCIETY

Volume 135, Number 8, August 2007, Pages 2391-2401

S 0002-9939(07)08763-1

Article electronically published on March 29, 2007

\title{
ON ZEROS OF EISENSTEIN SERIES FOR GENUS ZERO FUCHSIAN GROUPS
}

\author{
HEEKYOUNG HAHN
}

(Communicated by Ken Ono)

\begin{abstract}
Let $\Gamma \leq \mathrm{SL}_{2}(\mathbb{R})$ be a genus zero Fuchsian group of the first kind with $\infty$ as a cusp, and let $E_{2 k}^{\Gamma}$ be the holomorphic Eisenstein series of weight $2 k$ on $\Gamma$ that is nonvanishing at $\infty$ and vanishes at all the other cusps (provided that such an Eisenstein series exists). Under certain assumptions on $\Gamma$, and on a choice of a fundamental domain $\mathcal{F}$, we prove that all but possibly $c(\Gamma, \mathcal{F})$ of the nontrivial zeros of $E_{2 k}^{\Gamma}$ lie on a certain subset of $\left\{z \in \mathfrak{H}: j_{\Gamma}(z) \in \mathbb{R}\right\}$. Here $c(\Gamma, \mathcal{F})$ is a constant that does not depend on the weight, $\mathfrak{H}$ is the upper half-plane, and $j_{\Gamma}$ is the canonical hauptmodul for $\Gamma$.
\end{abstract}

\section{Introduction AND STATEMENT OF THE RESUlt}

Recently, Rudnick [10] showed that, under the Generalized Riemann Hypothesis, the zeros of Hecke eigenforms for $\mathrm{SL}_{2}(\mathbb{Z})$ become equidistributed with respect to the hyperbolic measure on a fundamental domain for the action of $\mathrm{SL}_{2}(\mathbb{Z})$. However, the situation is quite different in the case of zeros of Eisenstein series for $\mathrm{SL}_{2}(\mathbb{Z})$. F. Rankin and Swinnerton-Dyer [8] proved that all the zeros of Eisenstein series of even weight $k \geq 4$ for $\mathrm{SL}_{2}(\mathbb{Z})$ lie only on the arc $\left\{e^{i \theta} \mid \pi / 2 \leq \theta \leq 2 \pi / 3\right\}$ in the standard fundamental domain. R. Rankin 9] proved that similar properties hold for certain Poincaré series on $\mathrm{SL}_{2}(\mathbb{Z})$. Getz 4 generalized Rankin and SwinnertonDyer's result by providing conditions under which the zeros of other modular forms lie on this arc. Given these works, it is natural to investigate the behavior of the zeros of families of modular forms (in particular, the family of even weight Eisenstein series) on other groups. The goal of the present paper is to find the location of the zeros of certain Eisenstein series on genus zero Fuchsian groups.

From the outset we denote by $\Gamma$ a Fuchsian group of the first kind $[12$, by $\mathfrak{H}$ the upper half-plane, and by $\mathfrak{H}^{*}$ the union of $\mathfrak{H}$ with the set of cusps of $\Gamma$. We say that a meromorphic function $f$ on $\mathfrak{H}$ is a meromorphic modular form of weight $k$ for $\Gamma$ if

$$
f\left(\frac{a z+b}{c z+d}\right)=(c z+d)^{k} f(z)
$$

for all $z \in \mathfrak{H}$ and $\left(\begin{array}{ll}a & b \\ c & d\end{array}\right) \in \Gamma$, and $f$ is meromorphic at the cusps. If $k=0$, then $f$ is known as a modular function on $\Gamma$. Furthermore, we say that $f$ is a holomorphic

Received by the editors March 21, 2006 and, in revised form, April 27, 2006.

2000 Mathematics Subject Classification. Primary 11F03, 11F11.

Key words and phrases. Eisenstein series, modular forms, divisor polynomials.

This research was supported in part by a National Science Foundation FRG grant (DMS 0244660).

(C)2007 American Mathematical Society Reverts to public domain 28 years from publication 
modular form if $f$ is holomorphic on $\mathfrak{H}$ and holomorphic at the cusps. A holomorphic modular form is said to be a cusp form if it vanishes at the cusps of $\Gamma$. As usual, we denote by $M_{2 k}(\Gamma)$ (resp. $S_{2 k}(\Gamma)$ ) the space of holomorphic modular forms (resp. cusp forms) of weight $2 k$ for $\Gamma$. Moreover denote by $M_{2 k}^{\infty}(\Gamma)$ the space of weakly holomorphic modular forms on $\Gamma$ (i.e., holomorphic on $\mathfrak{H}$ but not necessarily at the cusps).

If $\Gamma$ has a cusp at $\infty$ with width $h$, then each $f \in M_{2 k}^{\infty}(\Gamma)$ has a Fourier expansion at infinity:

$$
f(z)=\sum_{n=n_{0}}^{\infty} a_{n} q_{h}^{n}, \quad q_{h}:=e^{2 \pi i z / h}, \quad n_{0} \in \mathbb{Z} .
$$

Note that a modular form can be identified with its $q$-expansion. We say that the form has real Fourier coefficients if $a_{n}$ is real for all $n$.

Assume that $\Gamma$ has $\infty$ as a cusp. Let $E_{2 k}^{\Gamma} \in M_{2 k}(\Gamma)$ be the unique modular form that is orthogonal to all cusp forms, vanishes at all cusps but $\infty$ and has constant term 1 in its Fourier expansion at $\infty$ (provided that such a modular form exists). We will refer to $E_{2 k}^{\Gamma}$ as the Eisenstein series associated to the cusp $\infty$. This is the Eisenstein series to which we referred in the title of this paper.

Remark. Note that such a form doesn't always exist. For instance, take $k=1$ and $\Gamma=\mathrm{SL}_{2}(\mathbb{Z})$.

Definition. The group $\Gamma$ is good for the weight $2 k$ if

(1) the Eisenstein series $E_{2 k}^{\Gamma}$ (if it exists) has real Fourier coefficients;

(2) the space $M_{2 k}(\Gamma)$ has a basis of forms with real Fourier coefficients.

Remark. For example, $\Gamma_{0}(N)$ is good for all $N$. See $\$ 2$,

Assume $\Gamma$ has genus zero. In this case, it is well known that there is a unique weakly holomorphic modular function with a Fourier expansion of the following form:

$$
j_{\Gamma}(z)=\frac{1}{q_{h}}+\sum_{n=1}^{\infty} a_{n} q_{h}^{n} \in M_{0}^{\infty}(\Gamma) .
$$

This function $j_{\Gamma}$ is referred to as the canonical hauptmodul.

Suppose $\Gamma$ has a cusp at $\infty$ with width $h$. We choose a simply connected fundamental domain $\mathcal{F}$ for $\Gamma$ contained in $\{z:|\Re(z)| \leq h / 2\}$. Define $y_{0} \in \mathbb{R}$ by

$$
y_{0}:=\inf \{y: \pm h / 2+i y \in \partial \mathcal{F}\},
$$

where $\partial \mathcal{F}$ denotes the boundary of $\mathcal{F}$. We refer to the complement (in $\partial \mathcal{F}$ ) of the vertical lines $\left\{z: \Re(z)= \pm h / 2\right.$, and $\left.\Im(z)>y_{0}\right\}$ as the lower arcs $A$.

For the purposes of our paper, we need to place some restrictions on our fundamental domain. Assume that we can parametrize $A$ by a piecewise smooth function

$$
\begin{aligned}
z_{A}=x_{A}+i y_{A}:(0,1) & \longrightarrow A \\
t & \longmapsto z_{A}(t) .
\end{aligned}
$$

Fixing such a parametrization, consider the set of points

$$
\operatorname{Crit}(\mathcal{F}):=\left\{t \in(0,1): \frac{y^{\prime}(t)}{\left(j_{\Gamma} \circ z_{A}\right)^{\prime}(t)}=0 \text { or } \frac{y^{\prime}(t)}{\left(j_{\Gamma} \circ z_{A}\right)^{\prime}(t)} \text { is undefined }\right\} .
$$


Here the ' indicates differentiation with respect to $t$. We say that $\frac{y^{\prime}(t)}{\left(j_{\Gamma} \circ z_{A}\right)^{\prime}(t)}$ changes sign at $t_{0}$ if, for any sufficiently small $\epsilon_{1}, \epsilon_{2}>0$,

$$
\left(\frac{y^{\prime}\left(t_{0}-\epsilon_{1}\right)}{\left(j_{\Gamma} \circ z_{A}\right)^{\prime}\left(t_{0}-\epsilon_{1}\right)}\right)\left(\frac{y^{\prime}\left(t_{0}+\epsilon_{2}\right)}{\left(j_{\Gamma} \circ z_{A}\right)^{\prime}\left(t_{0}+\epsilon_{2}\right)}\right)<0
$$

We make the following definition:

Definition. A fundamental domain $\mathcal{F}$ for $\Gamma$ is acceptable if we can parametrize $A$ by a piecewise smooth path $z_{A}$ as above and the following conditions hold:

(1) The hauptmodul $j_{\Gamma}$ is real on $\partial \mathcal{F}$.

(2) $\operatorname{Crit}(\mathcal{F})$ is a discrete set.

(3) If $\gamma z_{A}\left(t_{0}\right)=z_{A}\left(\hat{t}_{0}\right)$ for some $\gamma \in \Gamma$ and for some $t_{0}, \hat{t}_{0} \in \operatorname{Crit}(\mathcal{F})$, then $\frac{y^{\prime}(t)}{\left(j_{\Gamma} \circ z_{A}\right)^{\prime}(t)}$ changes sign at $t_{0}$ if and only if it changes sign at $\hat{t}_{0}$.

Remark. These appear to be natural restrictions on $\mathcal{F}$. In $₫ 2$ we deal with the case $\Gamma=\Gamma_{0}(3)$, for example. We must point out, however, that we do not have a proof that such a fundamental domain exists for an arbitrary $\Gamma$ of good genus zero.

Now, define

$$
c(\Gamma, \mathcal{F}):=\mid\left\{z_{A}\left(t_{0}\right): t_{0} \in \operatorname{Crit}(\mathcal{F}) \text { and } \frac{y^{\prime}(t)}{\left(j_{\Gamma} \circ z_{A}\right)^{\prime}(t)} \text { changes sign at } t_{0}\right\} / \sim \mid,
$$

where we consider two points in the set to be equivalent if they are $\Gamma$-conjugates of each other. Note that $c(\Gamma, \mathcal{F})$ depends on $\Gamma$ and on a choice of $\mathcal{F}$, but not on the weight. For each $f \in M_{2 k}(\Gamma)$, let

$$
P(f, X):=\prod_{\substack{Q \in \mathcal{F} \backslash\{\infty\} \\ \operatorname{ord}_{Q} f \neq 0}}\left(X-j_{\Gamma}(Q)\right)^{\operatorname{ord}_{Q}(f)-e_{Q}\left\{k\left(1-1 / e_{Q}\right)\right\}} .
$$

Here $\left\{k\left(1-1 / e_{Q}\right)\right\}$ is the fractional part of $k\left(1-1 / e_{Q}\right)$, and $e_{Q}:=\left|\operatorname{Stab}_{\bar{\Gamma}}(Q)\right|$, where $\bar{\Gamma}:=\Gamma /\{ \pm I\}$ if $-I \in \Gamma$ and $\bar{\Gamma}=\Gamma$ otherwise. Here $I$ is the identity matrix. Thus if $P\left(f, j_{\Gamma}(Q)\right)=0$, then $f(Q)=0$, with the converse being true if $Q$ is not an elliptic fixed point. It follows from Lemma 3.1 below that $P(f, X) \in \mathbb{C}[X]$ (this is obvious if the divisor of $f$ contains no elliptic fixed points). We call $P(f, X)$ the divisor polynomial of $f$. Let

$$
a_{0}:=j_{\Gamma}\left(-h / 2+i y_{0}\right),
$$

where $y_{0}$ is defined as in (1.3). We can now state our main theorem:

Theorem 1.1. Let $\Gamma$ be a genus zero group that is good for the weight $2 k$. Suppose that $\mathcal{F}$ is acceptable for $\Gamma$ and that $j_{\Gamma}$ has real Fourier coefficients. Then all but possibly $c(\Gamma, \mathcal{F})$ of the zeros of $P\left(E_{2 k}^{\Gamma}, X\right)$ lie on $\left[a_{0}, \infty\right)$, where $a_{0}$ is as in (1.5). Moreover, if $m$ denotes the number of distinct zeros with odd multiplicity on $\left[a_{0}, \infty\right)$, then $m+c(\Gamma, \mathcal{F}) \geq \operatorname{deg}\left(P\left(E_{2 k}^{\Gamma}, X\right)\right)$.

Remark. Since $c(\Gamma, \mathcal{F})$ is very small, Theorem 1.1 implies that almost all zeros of $P\left(E_{2 k}^{\Gamma}, X\right)$ are simple. For example, in the case $\Gamma=\mathrm{SL}_{2}(\mathbb{Z})$ and $\mathcal{F}$ is the standard fundamental domain, we have $c(\Gamma, \mathcal{F})=0$ (see \$2), and therefore all the zeros of $P\left(E_{2 k}^{\Gamma}, X\right)$ are simple and lie on $[0, \infty)$. Thus in this case our result is weaker than Rankin and Swinnerton-Dyer's result [8], but it has the advantage that the same argument applies to a family of groups. 
Recall the elementary fact that the complex zeros of a polynomial with real coefficients must occur in pairs. With this in mind, the alternate construction of $P(f, X)$ given in (3.2) below immediately implies the following corollary of Theorem 1.1 .

Corollary 1.2. Assume the hypotheses of Theorem 1.1. If $c(\Gamma, \mathcal{F})$ is odd, then all but possibly $c(\Gamma, \mathcal{F})-1$ of the zeros of $P\left(E_{2 k}^{\Gamma}, X\right)$ are real.

The proof of Theorem 1.1 is given in 4 . It can be viewed as an adaptation of the classical argument proving that the zeros of an orthogonal polynomial are all real and simple (see [1, Theorem 5.4.1], for example). We now outline the proof. Let $d:=\operatorname{dim}\left(M_{2 k}(\Gamma)\right)$. Using the Riemann-Roch theorem, we first construct a modular form $\Upsilon \in M_{2 k}(\Gamma)$ with a zero of order $d-1$ at infinity and no other "nontrivial" zeros. Suppose for a contradiction that the result is not true. Then we can construct a polynomial $Q(X)$ of degree less than $d-1$ whose zeros are exactly the zeros of $P\left(E_{2 k}^{\Gamma}, X\right)$ that occur with odd multiplicity and the image under $j_{\Gamma}$ of points $z_{A}(t)$, where $\frac{y^{\prime}(t)}{\left(j_{\Gamma} \circ z_{A}\right)^{\prime}(t)}$ changes sign along the lower arcs $A$. Then $Q\left(j_{\Gamma}\right) \Upsilon$ will be an element of $M_{2 k}(\Gamma)$ that vanishes at $\infty$, and it follows that the Petersson inner product $\left\langle E_{2 k}^{\Gamma}, Q\left(j_{\Gamma}\right) \Upsilon\right\rangle$ is zero. On the other hand, using Green's theorem, we relate the Petersson inner product $\left\langle E_{2 k}^{\Gamma}, Q\left(j_{\Gamma}\right) \Upsilon\right\rangle$ to a line integral along the lower arcs. By our construction of $Q(X)$, we show that this line integral cannot be zero, resulting in the desired contradiction.

We close this introduction with the following remark:

Remark. Suppose that a genus zero $\Gamma$ is good and admits an acceptable fundamental domain. Since $\Gamma$ is of genus zero, $j_{\Gamma}$ defines a model $j_{\Gamma}: \Gamma \backslash \mathfrak{H}^{*} \rightarrow \mathbb{P}^{1}(\mathbb{C})$ of $\Gamma \backslash \mathfrak{H}^{*}$ as the Riemann sphere. One can interpret Theorem 1.1 as saying that all but a bounded number of the zeros of $E_{2 k}^{\Gamma}$ lie on the real points of this model. It would be interesting to see if some modification of the ideas of this paper could prove that, for suitable higher genus congruence subgroups $\Gamma$, some proportion of the zeros of corresponding Eisenstein series lie either at the Weierstrass points or the real points of a suitable model for $\Gamma \backslash \mathfrak{H}^{*}$. We should emphasize that this is only a guess as to what occurs in these cases; we have not even done any numerical computations.

\section{EXAmples}

In this section, we give some examples of good groups and acceptable fundamental domains. We note that we found the fundamental domain drawing applet [13] useful in producing these examples.

Clearly $\mathrm{SL}_{2}(\mathbb{Z})$ is good. The principal congruence subgroup $\Gamma(N)$ of $\mathrm{SL}_{2}(\mathbb{Z})$,

$$
\Gamma(N):=\left\{\gamma \in \mathrm{SL}_{2}(\mathbb{Z}): \gamma \equiv\left(\begin{array}{ll}
1 & 0 \\
0 & 1
\end{array}\right) \quad(\bmod N)\right\}
$$

is good for all $2 k$ [11, p.163]. For an integer $N \geq 1$, any group $\Gamma$ satisfying $\Gamma_{1}(N) \leq$ $\Gamma \leq \Gamma_{0}(N)$ is good 3 ] for all $2 k$, where

$$
\begin{aligned}
& \Gamma_{1}(N):=\left\{\gamma \in \mathrm{SL}_{2}(\mathbb{Z}): \gamma \equiv\left(\begin{array}{ll}
1 & * \\
0 & 1
\end{array}\right) \quad(\bmod N)\right\}, \\
& \Gamma_{0}(N):=\left\{\gamma \in \mathrm{SL}_{2}(\mathbb{Z}): \gamma \equiv\left(\begin{array}{ll}
* & * \\
0 & *
\end{array}\right) \quad(\bmod N)\right\} .
\end{aligned}
$$


Define the group $\Gamma_{0}^{*}(N) \leq \mathrm{SL}_{2}(\mathbb{R})$ as in [12, p.27] to be the extension of $\Gamma_{0}(N)$ by the Fricke involution,

$$
W_{N}:=\left(\begin{array}{cc}
0 & -\sqrt{N}^{-1} \\
\sqrt{N} & 0
\end{array}\right) .
$$

Since $\Gamma_{0}(N)$ is good for all $2 k$ and an involution has eigenvalues \pm 1 , there is a basis of eigenvectors for $W_{N}$ acting on $M_{2 k}\left(\Gamma_{0}(N)\right)$ whose Fourier coefficients are all real. The forms in this basis with eigenvalue +1 will be a basis for $M_{2 k}\left(\Gamma_{0}^{*}(N)\right)$; it follows that the $\Gamma_{0}^{*}(N)$ are good.

Some good groups have acceptable fundamental domains. Before we consider any examples, we point out that if $j_{\Gamma}$ has real Fourier coefficients, then it is always real on $\Re(z)= \pm \frac{h}{2}$, where $h$ is the width of the cusp at $\infty$. This is easy to see from its Fourier expansion at $\infty$.

We now consider a few examples. If $\Gamma=\mathrm{SL}_{2}(\mathbb{Z})$, the classical fundamental domain bounded by $\Re(z)= \pm \frac{1}{2}$ and the unit circle is acceptable [11, p.32], and it is clear that $c(\Gamma, \mathcal{F})=0$ since $\frac{\partial y}{\partial j_{\Gamma}}$ does not change sign on $\left\{e^{i \theta} \mid \frac{\pi}{2} \leq \theta \leq \frac{2 \pi}{3}\right\}$.

Consider $\Gamma=\Gamma_{0}(3)$. In this case, the Eisenstein series $E_{2 k}^{\Gamma}(k \geq 1)$, has real Fourier coefficients (see [2, Theorem 1.1]). Denote by $\mathcal{F}$ the fundamental domain bounded by vertical lines $\left\{z: \Re(z)= \pm \frac{1}{2}\right.$, and $\left.\Im(z)>\frac{\sqrt{3}}{6}\right\}, A_{l}=\left\{z:-\frac{1}{2} \leq \Re(z) \leq\right.$ 0 and $\left.\left|z+\frac{1}{3}\right|=\frac{1}{3}\right\}$, and $A_{r}=\left\{z: 0 \leq \Re(z) \leq \frac{1}{2}\right.$ and $\left.\left|z-\frac{1}{3}\right|=\frac{1}{3}\right\}$. The canonical hauptmodul $j_{3}(z)$ for $\Gamma_{0}(3)$ has real Fourier coefficients (see [7, p. 317]). This implies that $j_{3}(z)=\overline{j_{3}(-\bar{z})}$. For $z \in A_{l}$, a simple calculation shows that $\gamma z=-\bar{z}$, where

$$
\gamma:=\left(\begin{array}{cc}
1 & 0 \\
-3 & 1
\end{array}\right) \in \Gamma_{0}(3)
$$

and hence $j_{3}(z)=\overline{j_{3}(-\bar{z})}=\overline{j_{3}(\gamma z)}=\overline{j_{3}(z)}$, because $j_{3}$ is invariant under $\Gamma_{0}(3)$. Similarly, for $z \in A_{r}$, we have $\gamma^{\prime} z=-\bar{z}$, where

$$
\gamma^{\prime}:=\left(\begin{array}{ll}
1 & 0 \\
3 & 1
\end{array}\right) \in \Gamma_{0}(3) .
$$

Therefore we have $j_{3}(z) \in \mathbb{R}$ for all $z \in \partial \mathcal{F}$. Since $c(\Gamma, \mathcal{F})=1$ in this case, all the zeros of $P\left(E_{2 k}^{\Gamma}, X\right)$ are simple, and all but possibly one of the zeros lie on $[-42, \infty)$. Here we used the fact that $j_{3}\left(\frac{-3+i \sqrt{3}}{6}\right)=-42$ (see [7. p. 317]).

\section{Preliminaries on Divisor POLYNOMIALS}

In this section we prove a proposition which gives an alternative construction of the divisor polynomial $P(f, X)$ attached to a modular form $f \in M_{2 k}(\Gamma)$. The idea is basically that of Ono (see [6. Section 2.6]), but things are complicated in our case due to the fact that the algebra structure of $\bigoplus_{k \geq 0} M_{2 k}(\Gamma)$ is not always as simple as it is in the case $\Gamma=\mathrm{SL}_{2}(\mathbb{Z})$. To overcome this difficulty, we rely on a few easy applications of Riemann-Roch theory. As above, for $Q \in \mathfrak{H}^{*}$, we define $e_{Q}:=\left|\operatorname{Stab}_{\bar{\Gamma}}(Q)\right|$, where $\bar{\Gamma}$ is the image of $\Gamma$ in $\operatorname{PSL}_{2}(\mathbb{R})$.

Lemma 3.1. Any modular form in $M_{2 k}(\Gamma)$ has a zero of order at least $e_{Q}\{k(1-$ $\left.\left.1 / e_{Q}\right)\right\}$ at every elliptic fixed point $Q \in \mathfrak{H}^{*}$.

Proof. Let $f \in M_{2 k}(\Gamma)$ and let $\omega$ be the corresponding $k$-fold differential form on $\Gamma \backslash \mathfrak{H}^{*}$. If $Q$ is an elliptic fixed point, then

$$
\operatorname{ord}_{Q}(f)=e_{Q} \operatorname{ord}_{P}(\omega)+k\left(e_{Q}-1\right) .
$$


Here $P=\pi(Q)$, where $\pi: \mathfrak{H}^{*} \rightarrow \Gamma \backslash \mathfrak{H}^{*}$ is the canonical projection (see [5, Lemma 4.11]). Since $Q$ is elliptic, it is well known that $e_{Q}=2$ or 3 . In view of this, it is easy to check that (3.1) implies the lemma.

Using Lemma 3.1 we now prove the following proposition:

Proposition 3.2. Let $d:=\operatorname{dim}\left(M_{2 k}(\Gamma)\right)$. If $\Gamma$ is of genus zero, then there exists a modular form $\Upsilon \in M_{2 k}(\Gamma)$ uniquely characterized by the following conditions:

(1) The Fourier expansion of $\Upsilon$ at $\infty$ is of the form $q_{h}^{d-1}+\sum_{n>d} a_{n} q_{h}^{n}$.

(2) For every elliptic fixed point $Q \in \mathfrak{H}$, we have $\operatorname{ord}_{Q}(\Upsilon)=e_{Q}\left\{k\left(1-1 / e_{Q}\right)\right\}$.

(3) At every cusp of $\Gamma$ not equivalent to $\infty, \Upsilon$ does not vanish.

Moreover, if $\Gamma$ is good for the weight $2 k$, then $\Upsilon$ has real Fourier coefficients.

We defer the proof of Proposition 3.2 to the end of the section. Assuming it for the moment, we claim that if $f \in M_{2 k}(\Gamma)$ has 1 as the leading term of its Fourier expansion at $\infty$, then

$$
f \Upsilon^{-1}=P\left(f, j_{\Gamma}\right),
$$

where $P(f, X)$ is the divisor polynomial of $f$, defined as in (1.4). Indeed, by Proposition 3.2, $f \Upsilon^{-1} \in M_{0}^{\infty}(\Gamma)$ and has the zeros specified by (1.4) to the correct order. Since every element of $M_{0}^{\infty}(\Gamma)$ is a polynomial in $j_{\Gamma}$, the claim follows.

In the following proof, we use standard notation from Riemann-Roch theory on algebraic curves. See ([5, pp. 15-17]), for example.

Proof of Proposition 3.2. Since $\Gamma$ has genus zero, we have an isomorphism

$$
j_{\Gamma}: \Gamma \backslash \mathfrak{H}^{*} \stackrel{\sim}{\longrightarrow} \mathbb{P}^{1}(\mathbb{C}),
$$

so we may view $j_{\Gamma}$ as a coordinate for the Riemann sphere. Define the divisor $D$ by

$$
D=\operatorname{div}\left(\left(d j_{\Gamma}\right)^{k}\right)+(1-d+k) P_{\infty}+\sum_{\substack{\text { cusps } P \\ P \neq \infty}} k P+\sum_{\text {elliptic } P}\left[k\left(1-1 / e_{P}\right)\right] P,
$$

where $P_{\infty}$ is the point "infinity," and $\left[k\left(1-1 / e_{P}\right)\right]$ is the integer part of $k\left(1-1 / e_{P}\right)$, and if $\pi(Q)=P$ for some elliptic fixed point $Q$, then $e_{P}=e_{Q}$.

Let $\omega=f \cdot\left(d j_{\Gamma}\right)^{k}$ be a meromorphic differential $k$-form on $\Gamma \backslash \mathfrak{H}^{*}$. First we claim that $\omega$ corresponds to a nonzero constant multiple of a holomorphic modular form satisfying the conditions (1), (2) and (3) if and only if $f \in L(D)$. Let $f \in L(D)$. Then we have $\operatorname{div}(f)+D \geq 0$, and hence,

$$
\begin{aligned}
\operatorname{ord}_{P}(\omega)+k\left(1-1 / e_{P}\right) & \geq 0 \text { at the image of an elliptic fixed point, } \\
\operatorname{ord}_{P}(\omega)+k & \geq 0 \text { at the image of a cusp, } \\
\operatorname{ord}_{P}(\omega) & \geq 0 \text { at the remaining points. }
\end{aligned}
$$

It is known [5, Lemma 4.11] that if $\pi(Q)=P$ under the canonical projection $\pi: \mathfrak{H}^{*} \rightarrow \Gamma \backslash \mathfrak{H}^{*}$, then the following are true:

$$
\begin{aligned}
& \operatorname{ord}_{Q}(f)=e_{Q} \operatorname{ord}_{P}(\omega)+k\left(e_{Q}-1\right) \text { at an elliptic fixed point, } \\
& \operatorname{ord}_{Q}(f)=\operatorname{ord}_{P}(\omega)+k \text { at a cusp, } \\
& \operatorname{ord}_{Q}(f)=\operatorname{ord}_{P}(\omega) \text { at the remaining points. }
\end{aligned}
$$


Therefore $\operatorname{ord}_{Q}(f) \geq 0$ for all $Q \in \mathfrak{H}^{*}$, and so $f$ is holomorphic. Clearly, at $\infty$, we have $\operatorname{ord}_{P}(\omega)=d-1-k$, and hence $\operatorname{ord}_{Q}(f)=d-1$. It is also known [12, p. 46] that

$$
\sum\left(\operatorname{ord}_{Q}(f) / e_{Q}-k\left(1-1 / e_{Q}\right)\right)=-2 k+\nu_{\infty} k
$$

and

$$
d=1-2 k+\nu_{\infty} k+\sum\left[k\left(1-1 / e_{Q}\right)\right],
$$

where $\nu_{\infty}$ is the number of inequivalent cusps. Since $f$ has a zero of order $d-1$ at $\infty$, by comparing (3.4) with (3.5), we can conclude that $f$ has a zero of order $e_{Q}\left\{k\left(1-1 / e_{Q}\right)\right\}$ at each elliptic fixed point $Q$. Moreover, $f$ has no other zeros on $\mathfrak{H}$ and does not vanish at any cusp of $\Gamma$ inequivalent to $\infty$.

Secondly, we want to show that $l(D)=1$. Clearly

$$
\operatorname{deg}(D)=-2 k+(1-d+k)+\left(\nu_{\infty}-1\right) k+\sum\left[k\left(1-1 / e_{P}\right)\right] .
$$

By (3.5), we then obtain that $\operatorname{deg}(D)=0$. Hence by the Riemann-Roch theorem, we derive that $l(D)=1$. Therefore there is a unique $\Upsilon \in M_{2 k}(\Gamma)$ satisfying (1), (2) and (3).

We are left with proving that if $\Gamma$ is good, then $\Upsilon$ has real Fourier coefficients. Notice that (3.4) and (3.5), together with Lemma 3.1, imply that any modular form in $M_{2 k}(\Gamma)$ has order less than or equal to $d-1$ at $\infty$. In view of what we have proved above combined with Lemma 3.1, $\Upsilon$ is uniquely characterized by the facts that

(1) the leading term in the Fourier expansion of $\Upsilon$ at $\infty$ is 1 , and

(2) any $f \in M_{2 k}(\Gamma)$ that is not a scalar multiple of $\Upsilon$ satisfies $\operatorname{ord}_{\infty}(f)<$ $\operatorname{ord}_{\infty}(\Upsilon)$.

These conditions can be rephrased as saying that among the elements of $M_{2 k}(\Gamma)$ with leading term 1 in their Fourier expansion at $\infty, \Upsilon$ has the maximum number of zeros at $\infty$ and its Fourier expansion is $q_{h}^{d-1}+O\left(q_{h}^{d}\right)$. Since $M_{2 k}(\Gamma)$ has a basis of forms with real Fourier coefficients, it follows from simple linear algebra that $\Upsilon$ is a real linear combination of forms in this basis, and hence itself has real Fourier coefficients.

\section{Proof of Theorem 1.1}

We begin this section by recalling the Petersson inner product. If $f$ and $g$ are two modular forms in $M_{2 k}(\Gamma)$, then their Petersson inner product is defined by

$$
\langle f, g\rangle=\iint_{\mathcal{F}} f(z) \overline{g(z)} y^{2 k-2} d x d y,
$$

where $\mathcal{F}$ denotes a fundamental domain for the action of $\Gamma$, and where $z=x+i y$. It is well known that if $f g$ vanishes at every cusp of $\Gamma$, then the integral (4.1) converges. In Section 3 we showed that there is a divisor polynomial $P\left(f, j_{\Gamma}\right)\left(\right.$ resp. $\left.P\left(g, j_{\Gamma}\right)\right)$ attached to $f$ (resp. $g$ ) such that $f=P\left(f, j_{\Gamma}\right) \Upsilon\left(\right.$ resp. $\left.g=P\left(g, j_{\Gamma}\right) \Upsilon\right)$. So we derive that

$$
\langle f, g\rangle=\iint_{\mathcal{F}} P\left(f, j_{\Gamma}(z)\right) \overline{P\left(g, j_{\Gamma}(z)\right)}|\Upsilon(z)|^{2} y^{2 k-2} d x d y
$$


Suppose that $\Gamma$ admits an acceptable fundamental domain $\mathcal{F}$, and let $A$ be the lower arc of $\mathcal{F}$ as defined in $₫ 1$. Write

$$
A=\amalg_{i=1}^{n}\left\{A_{i}\right\},
$$

where the $A_{i}$ are smooth (rectifiable) paths such that $\left.j_{\Gamma}\right|_{\bar{A}_{i}}$ is injective. We then write $\overline{\mathcal{F}}=\bigcup_{i=1}^{n+2} \mathcal{F}_{i}$ where the $\mathcal{F}_{i}$ are the closures of domains in $\mathfrak{H}$ of positive area with respect to the hyperbolic metric that satisfy the following properties:

(1) If $i \neq j, \mathcal{F}_{i} \cap \mathcal{F}_{j}$ is a smooth curve that is rectifiable with respect to the hyperbolic metric.

(2) For $1 \leq i \leq n$ we have $A_{i} \subset \mathcal{F}_{i}$, and $A_{i} \cap \mathcal{F}_{j}$ is either empty or a single point for $i \neq j$.

(3) $\partial \mathcal{F}_{i}-\overline{A_{i}}$ is contained in the interior of $\mathcal{F}$.

(4) $\mathcal{F}_{n+1}=\overline{\mathcal{F}} \cap\{z: \Re(z) \leq 0\} \cap\left\{z: \Im(z) \geq y_{0}\right\}$ and $\mathcal{F}_{n+2}=\overline{\mathcal{F}} \cap\{z: \Re(z) \geq$ $0\} \cap\left\{z: \Im(z) \geq y_{0}\right\}$, where $y_{0}$ is defined as in (1.3).

(5) $\left.j_{\Gamma}\right|_{\mathcal{F}_{i}}$ is injective.

One can always write $\overline{\mathcal{F}}$ as such a union; we do this to avoid pathologies with certain line integrals, which we will now introduce.

For $z=x+i y \in \mathcal{F}_{i}$, define a path $\gamma_{i}(z)$ by

$$
\gamma_{i}(z):=\left\{j_{\Gamma}^{-1}\left(\xi+i \Im\left(j_{\Gamma}(z)\right)\right): \xi \in\left[\Re\left(j_{\Gamma}(z)\right), \infty\right)\right\}
$$

traversed from $\infty$ to $z$. Let $\Phi_{i}(z)$ be the path integral

$$
\Phi_{i}(z):=\int_{\gamma_{i}(z)} \Re\left(P\left(f, j_{\Gamma}(s)\right) \overline{P\left(g, j_{\Gamma}(s)\right)}|\Upsilon(s)|^{2} t^{2 k-2}\right) d t,
$$

where $\Im(s)=t$. Then we obtain the following result.

Proposition 4.1. Let $\Gamma$ be good for the weight $2 k$ of genus zero and $\mathcal{F}$ be an acceptable fundamental domain for $\Gamma$. Let $f$ and $g$ be modular forms in $M_{2 k}(\Gamma)$ such that $f g$ vanishes at every cusp of $\Gamma$. Defining $\mathcal{F}_{i}$ and $\Phi_{i}$ as above, we have that

$$
\iint_{\mathcal{F}} \Re\left(P\left(f, j_{\Gamma}(z)\right) \overline{P\left(g, j_{\Gamma}(z)\right)}|\Upsilon(z)|^{2} y^{2 k-2}\right) d x d y=-\sum_{i=1}^{n} \int_{A_{i}} \Phi_{i}(z) d x,
$$

where $z:=x+i y$, and the $A_{i}$ are the smooth paths in (4.2) traversed with counterclockwise orientation.

We will prove Proposition 4.1 after the proof of Theorem 1.1

Proof of Theorem 1.1. Let $\mathfrak{a}_{1}, \ldots, \mathfrak{a}_{m}$ be the zeros of $P\left(E_{2 k}^{\Gamma}, X\right)$ on $\left[a_{0}, \infty\right)$ that have odd multiplicity and let $\mathfrak{b}_{1}, \ldots, \mathfrak{b}_{c(\Gamma, \mathcal{F})}$ be the points along $\mathcal{F} \cap A$ where $\frac{\partial y}{\partial j_{\Gamma}}$ changes sign. Here $a_{0}$ is defined as in (1.5). If $m+c(\Gamma, \mathcal{F}) \geq \operatorname{deg}\left(P\left(E_{2 k}^{\Gamma}, X\right)\right)$, we are done, so we assume for a contradiction that $m+c(\Gamma, \mathcal{F})<\operatorname{deg}\left(P\left(E_{2 k}^{\Gamma}, X\right)\right)$. Let

$$
Q(X):=\prod_{i=1}^{m}\left(X-\mathfrak{a}_{i}\right) \prod_{j=1}^{c(\Gamma, \mathcal{F})}\left(X-j_{\Gamma}\left(\mathfrak{b}_{j}\right)\right) .
$$

Then $Q\left(j_{\Gamma}\right)$ is a polynomial in $j_{\Gamma}$ with real coefficients.

Note that $Q\left(j_{\Gamma}\right) \Upsilon \in M_{2 k}^{\infty}(\Gamma)$. In fact, by Lemma 3.2 and the construction of $Q(X)$, we have that $Q\left(j_{\Gamma}\right) \Upsilon \in M_{2 k}(\Gamma)$ and $Q\left(j_{\Gamma}\right) \Upsilon$ vanishes at $\infty$. Since any 
modular form in $M_{2 k}(\Gamma)$ that vanishes at infinity is orthogonal to $E_{2 k}^{\Gamma}$ with respect to the Petersson inner product, we have

$$
0=\left\langle E_{2 k}^{\Gamma}, Q\left(j_{\Gamma}\right) \Upsilon\right\rangle
$$

On the other hand, we have that for $z=x+i y$,

$$
\begin{aligned}
\left\langle E_{2 k}^{\Gamma}, Q\left(j_{\Gamma}\right) \Upsilon\right\rangle= & \iint_{\mathcal{F}} \Re\left(P\left(E_{2 k}^{\Gamma}, j_{\Gamma}(z)\right) \overline{Q\left(j_{\Gamma}(z)\right)}|\Upsilon(z)|^{2}\right) y^{2 k-2} d x d y \\
& +i \iint_{\mathcal{F}} \Im\left(P\left(E_{2 k}^{\Gamma}, j_{\Gamma}(z)\right) \overline{Q\left(j_{\Gamma}(z)\right)}|\Upsilon(z)|^{2}\right) y^{2 k-2} d x d y .
\end{aligned}
$$

By Proposition 4.1 we know that

$$
\begin{aligned}
& \iint_{\mathcal{F}} \Re\left(P\left(E_{2 k}^{\Gamma}, j_{\Gamma}(z)\right) \overline{Q\left(j_{\Gamma}(z)\right)}|\Upsilon(z)|^{2} y^{2 k-2}\right) d x d y \\
& \quad=-\sum_{i=1}^{n} \int_{A_{i}}\left(\int_{\infty}^{j_{\Gamma}(z)} P\left(E_{2 k}^{\Gamma}, j_{\Gamma}\right) Q\left(j_{\Gamma}\right)\left(|\Upsilon|^{2} t^{2 k}\right)\left(j_{\Gamma}\right) t^{-2}\left(j_{\Gamma}\right) t^{\prime}\left(j_{\Gamma}\right) d j_{\Gamma}\right) d x .
\end{aligned}
$$

Here we have used the fact that $\left.j_{\Gamma}\right|_{\mathcal{F}_{i}}$ is injective, so we may view $\Upsilon$ and $t$ as functions of $j_{\Gamma}$. Our path $\gamma_{i}(z)$ was chosen so that whenever $z \in A_{i}$, the function $j_{\Gamma}$ is real in $\left[j_{\Gamma}(z), \infty\right)$; we used this to change $\overline{Q\left(j_{\Gamma}\right)}$ to $Q\left(j_{\Gamma}\right)$ in (4.8). Moreover, by construction of $Q(X)$, we have that for each $z \in A_{i}, P\left(E_{2 k}^{\Gamma}, X\right) Q(X) t^{\prime}(X)$ is nonnegative or nonpositive on $\left[j_{\Gamma}(z), \infty\right)$, and its sign does not depend on $i$. It follows that the integral (4.8) cannot be zero. This is the desired contradiction.

We now give a proof of Proposition 4.1

Proof of Proposition 4.1. For $\delta \in\{1,2\}$, we define

$$
\mathcal{F}_{n+\delta}^{T}:=\mathcal{F}_{n+\delta} \cap\{z: \Im(z) \leq T\} \quad \text { and } \quad L_{n+\delta}^{T}=\mathcal{F}_{n+\delta}^{T} \cap\{z: \Im(z)=T\},
$$

for $T \gg 0$. Note that

$$
\frac{\partial \Phi_{i}(z)}{\partial y}=\Re\left(P\left(f, j_{\Gamma}(z)\right) \overline{P\left(g, j_{\Gamma}(z)\right)}|\Upsilon(z)|^{2} y^{2 k-2}\right) .
$$

We apply Green's theorem to each $\mathcal{F}_{i}$ separately, use (4.9), and then sum the contributions to obtain

$$
\begin{aligned}
& \iint_{\mathcal{F}} \Re\left(P\left(f, j_{\Gamma}(z)\right) \overline{P\left(g, j_{\Gamma}(z)\right)}|\Upsilon(z)|^{2} y^{2 k-2}\right) d x d y \\
& \quad=-\sum_{\delta=1}^{2} \lim _{T \rightarrow \infty} \int_{L_{n+\delta}^{T}} \Phi_{n+\delta}(z) d x-\sum_{i=1}^{n} \int_{A_{i}} \Phi_{i}(z) d x .
\end{aligned}
$$

Here the limit exists because $f g$ vanishes at $\infty$ (and hence has rapid decay). The contribution of the vertical lines contained in $\left\{z:|\Re(z)|= \pm \frac{h}{2}\right\} \cap \overline{\mathcal{F}}$ is zero because $d x=0$ there. We now claim that

$$
\lim _{T \rightarrow \infty} \int_{L_{n+\delta}^{T}} \Phi_{n+\delta}(z) d x=0 .
$$

It is easy to see that (4.11) in conjunction with (4.10) will finish the proof of the proposition. 
In view of the fact that $\int_{L_{n+\delta}^{T}} d x=h / 2<\infty$, in order to prove (4.11), it is enough to show that

$$
\Phi_{n+\delta}(z)=\int_{\gamma_{n+\delta}(z)} \Re\left(P\left(f, j_{\Gamma}(s)\right) \overline{P\left(g, j_{\Gamma}(s)\right)}|\Upsilon(s)|^{2} t^{2 k-2}\right) d t \rightarrow 0
$$

as $T \rightarrow \infty$ while $z \in L_{n+\delta}^{T}$. Here $s \in \gamma_{n+\delta}(z)$ and $t=\Im(s)$. Because $f g$ vanishes at every cusp, we have that

$$
\Re\left(P\left(f, j_{\Gamma}(s)\right) \overline{P\left(g, j_{\Gamma}(s)\right)}|\Upsilon(s)|^{2} t^{2 k}\right)=\Re\left(f(s) \overline{g(s)} t^{2 k}\right) \ll C
$$

for every $s \in \mathcal{F}$, where $C>0$ is some constant not depending on $s$. Therefore, to prove (4.12) it suffices to show that

$$
\int_{\gamma_{n+\delta}(z)} t^{-2} d t \rightarrow 0
$$

as $T \rightarrow \infty$ while $z \in L_{n+\delta}^{T}$. Note that since $j_{\Gamma}(x+i y) \approx e^{-2 \pi i(x+i y)}$ as $y \rightarrow \infty$, we have

$$
j_{\Gamma}^{-1}(x+i y) \approx \frac{1}{2 \pi}\left(i \log \sqrt{x^{2}+y^{2}}-\operatorname{Arg}(x+i y)\right),
$$

where $\log$ denotes the principal branch of the natural logarithm. So while $z \in L_{n+\delta}^{T}$,

$$
\begin{aligned}
\int_{\gamma_{n+\delta}(z)} t^{-2} d t & \approx \int_{\infty}^{\Re\left(j_{\Gamma}(z)\right)} \log {\sqrt{\xi^{2}+\Im\left(j_{\Gamma}(z)\right)^{2}}}^{-2}\left(\log {\left.\sqrt{\xi^{2}+\Im\left(j_{\Gamma}(z)\right)^{2}}\right)^{\prime} d \xi}^{\Re\left(j_{\Gamma}(z)\right)^{2}+\Im\left(j_{\Gamma}(z)\right)^{2}} \frac{d \xi}{\xi \log ^{2} \xi}\right. \\
& \approx \int_{\infty} \frac{1}{\log \left(\Re\left(j_{\Gamma}(z)\right)^{2}+\Im\left(j_{\Gamma}(z)\right)^{2}\right)} \rightarrow 0,
\end{aligned}
$$

as $T \rightarrow \infty$, since $\left|j_{\Gamma}(z)\right|^{2} \approx e^{4 \pi T}$ near $\infty$. This implies (4.13) and in turn the proposition.

\section{ACKNOWLEDGMENTS}

I am indebted to David Farmer, Jayce Getz, Steve Gonek and Ken Ono for their encouragement and helpful comments.

\section{REFERENCES}

1. G. E. Andrews, R. Askey, and R. Roy, Special functions, Encyclopedia of Mathematics 71, Cambridge Univ. Press, Cambridge, 1999. MR.1688958 (2000g:33001)

2. M. Boylan, Swinnerton-Dyer type congruences for certain Eisenstein series, Contemporary Mathematics 291 (2001), 93-108. MR1874523 (2002k:11063)

3. F. Diamond and J. Im, Modular forms and modular curves, Canadian Math. Soc. Conference Proceedings 17 (1995), 39-133. MR 1357209 (97g:11044)

4. J. Getz, A generalization of a theorem of Rankin and Swinnerton-Dyer on zeros of modular forms, Proc. American Math. Soc. 132 (2004), 2221-2231. MR2052397 (2005e:11047)

5. J. S. Milne, Modular functions and modular forms, Course note, http://www.jmilne.org/math, Univ. of Michigan, 1997.

6. K. Ono, The web of modularity: Arithmetic of the coefficients of modular forms and q-series, CBMS, 102, American Math. Soc., Providence, Rhode Island, 2004.

7. K. Ono and K. Bringmann, Identities for traces of singular moduli, Acta Arith. 119 (2005), 317-327.

8. F. K. C. Rankin and H. P. F. Swinnerton-Dyer, On the zeros of Eisenstein series, Bull. London Math. Soc. 2 (1970), 169-170. 
9. R. A. Rankin, The zeros of certain Poincaré series, Compositio Math. 46 (1982), 255-272. MR0664646 (83m:10036)

10. Z. Rudnick, On the asymptotic distribution of zeros of modular forms, IMRN, No. 34 (2005), 2059-2076. MR2181743

11. B. Schoeneberg, Elliptic modular functions: An introduction, Springer-Verlag, New York, Heidelberg, Berlin, 1974. MR0412107 (54:236)

12. G. Shimura, Introduction to the arithmetic theory of automorphic functions, Princeton Univ. Press, Princeton, 1971. MR0314766 (47:3318)

13. H. A. Verrill, Fundamental domain drawer, Java, http://www.math.lsu.edu/ ${ }^{\sim}$ verrill/ fundomain.

Department of Mathematics, University of Rochester, Rochester, New York 14627

E-mail address: hahn@math.rochester.edu 